\title{
The origin of bitumen and its significance of hydrocarbon accumulation
}

\author{
WANG ZHAOYUn, MA CHAO, GONG DEYU, JIANG HU
}

(Research Institute of Petroleum Exploration \& Development, PetroChina, Beijing 100083, China)

Bitumen in strata can be divided into primary and secondary bitumen according to the order of formation, and secondary bitumen can be divided into two genetic types of biodegradation-waterwashing-oxidationand and pyrolysis bitumen developed in different geological processes. This paper focuses on the discussion of pyrolysis bitumen. Pyrolysis bitumen is the residual from the cracking of oil as being further buried. Based on the study of more than 60 core samples from more than 10 wells in Sichuan Basin, the content of pyrolysis bitumen ranges between $0.1 \%-15 \%$, in the form of strip, sheet, block and powder, the reservoir rock is coarse-grained, medium-fine-grained, the occurrence modes are fracture (region connecting oil source), bedding surface, primary and secondary dissolution holes, microfractures, etc. The associated inclusions are rich, which can be divided into early and late filling of holes, secondary enlarged edge of dolomite, filling of calcite microcracks, etc., as well as tawny rich in bitumen inclusion. The color of inclusions is transparent colorless, light gray, light brown, dark gray, dark brown, etc. The homogenization temperature of inclusions ranges from $90{ }^{\circ} \mathrm{C}$ to $220{ }^{\circ} \mathrm{C}$, which comprehensively records the whole process of hydrocarbon generation and evolution, as well as the abnormal thermal action. The temperature distribution range of inclusions in the same sample is generally wide, reflecting the frequent dissolution and recrystallization of carbonate rocks. Based on the characteristics of pyrolysis bitumen and its reservoir, organic and inorganic geochemical analysis, inclusion study, as well as burial history, thermal history, oil and gas migration and accumulation process analysis, results show that the main source rocks of the ancient carbonate rocks in Sichuan Basin, including Qiongzhusi formation, section 1 \& 3 of Dengying formation, entered the threshold of oil generation at the end of Silurian, a large number of oil generated in the Middle Permian, the threshold of oil cracking and gas generated in the Late Permian, and a large amount of oil cracked into gas in the Late Triassic. Oil and gas have the characteristics of migration from north to south. The superimposition figure of pyrolysis bitumen and paleostructure is a key way to analyzing secondary reservoir formation.

Key words: pyrolysis bitumen; inorganic elements; inclusions; hydrocarbon accumulation 